\title{
Using MILP for UAVs Trajectory Optimization under Radar Detection Risk
}

\author{
José J. Ruz , Orlando Arévalo* \\ (*)Dept. of Computer Architecture and \\ Automatic Control \\ Universidad Complutense de Madrid \\ [jjruz, ojareval]@ fis.ucm.es
}

\begin{abstract}
This paper presents an approach to trajectories optimization for Unmanned Aerial Vehicle (UAV) in presence of obstacles, waypoints, and threat zones such as radar detection regions, using Mixed Integer Linear Programming (MILP). The main result is the linear approximation of a nonlinear radar detection risk function with integer constraints and indicator 0-1 variables. Several results are presented to show that the approach can yields trajectories depending on the acceptable risk of detection.
\end{abstract}

\section{Introduction}

An Unmanned Aerial Vehicle (UAV) is a powered aerial vehicle that does not carry a human operator. UAVs can be used to provide valuable information about an area of interest. In recent years, both military and civilian institutions have exhibited increased interest in the use of UAVs. Frequently such vehicles need minor human control from ground station because a detailed map of the environment is available ahead of time. One of the main threats for UAVs is radar guided surface to air missiles. Knowledge of a radar detection system and a good model of its capabilities can be used to create a UAV trajectory with acceptable risk. A good model for a radar detection system takes many factors into consideration [1]. The most basic is distance between the radar and the UAV. Another important factor is the Radar Cross Section (RCS) of the UAV exposed to the radar.

In this work, we approach the problem of finding an optimal trajectory to minimize the risk of being detected of UAVs by opponent radar detection systems. In addition, the aim is to find an optimal trajectory minimizing the total flight time between the base station and the final destination. Designing optimal trajectories for UAVs which reduces their detection against radar systems has been traditionally treated in the literature by avoidance of such areas. However, when the UAV must reach a target close to the radar, threat zones must be entered. To address such a situation we have used a model based on idealizing geometrical and physical UAV properties

\author{
Jesús M. de la Cruz ${ }^{*}$, Gonzalo Pajares ${ }^{* *}$ \\ $(* *)$ Dept. of Software Engineering and \\ Artificial Intelligence \\ Universidad Complutense de Madrid \\ [jmcruz, pajares]@fis.ucm.es
}

[2]. Trajectory optimization for UAVs in complex terrain with obstacles and threat zones is a difficult task due to the non-convex nature of problem. Our approach to optimal trajectory generation is based on Mixed Integer Linear Programming (MILP). Previous works demonstrated the use of MILP in trajectory design for vehicles under various technological constraints [3, 4]. MILP is a powerful mathematical programming framework that extends continuous linear programming to include integer decision variables. These variables allow the inclusion of nonlinear constraints and discrete decisions in the trajectory optimization. The aim of this paper is to approach the non-linear and non-separable terms of a radar detection function using MILP. Optimal solutions can be obtained for these trajectory generation problems using powerful software packages such as CPLEX [5, 6]. However, the computational complexity required grows with the length of the trajectory, the number of obstacles to be avoided and the threat zones. This limitation can be avoided by using MILP in a receding horizon framework $[7,8,9,10]$.

\section{System Model}

The UAV trajectory generation is represented as a 2D constraint optimization problem (Figure 1) in the $\mathrm{X}-\mathrm{Y}$ plane, characterized by a set of decision variables, a set of constraints and an objective function. The decision variables are the UAV state variables, i.e. position and speed. The constraints are derived from the interactions between the UAV and its environment. These include dynamics $\left(C_{D}\right)$, maximum turning force which causes a minimum turning radius $\left(C_{R}\right)$, rectangular obstacle avoidance $\left(C_{O}\right)$, and the reaching of the target $\left(C_{T}\right)$. The objective function includes different measures of the solution quality, although the most important is the minimization of the total flight time to reach the target. However, when there are threats that put in risk the UAV mission, for example missiles guided by radars, it becomes necessary to incorporate to the objective function some term that minimizes the risk of UAV detection $\left(R_{D}\right)$. Throughout this paper we assume that the UAV maintains a fixed altitude and the radar is on the ground. 


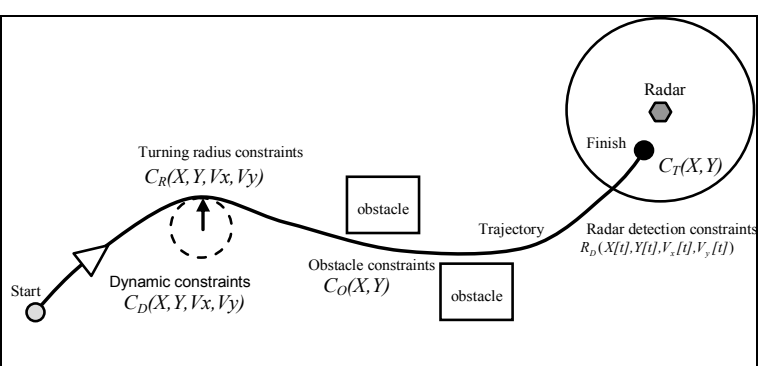

Figure 1. Trajectory generation by solving an optimization problem subject to constraints.

\subsection{Dynamic constraints}

The MILP trajectory optimization is constrained by a time-discrete dynamic which models the UAV with limitations in speed and turning rate. This is the consequence of a maximum magnitude in the turning force $\boldsymbol{u}(t)$ that the aircraft might be put under [9]. Dynamics is then expressed by Eqn.1.

$\left[\begin{array}{l}\vec{v}(t+1) \\ \vec{r}(t+1)\end{array}\right]=A \cdot\left[\begin{array}{l}\vec{v}(t) \\ \vec{r}(t)\end{array}\right]+B \cdot \vec{u}(t)$

$A$ and $B$ are the state matrices and $t$ the discrete time. Speed and force magnitudes are limited by the following constraints:

$$
\begin{aligned}
& v_{x} \cos \left(\varphi_{n}\right)+v_{y} \sin \left(\varphi_{n}\right) \leq\left|\vec{v}_{\max }\right| \\
& u_{x} \cos \left(\varphi_{n}\right)+u_{y} \sin \left(\varphi_{n}\right) \leq\left|\vec{u}_{\max }\right| \\
& \varphi_{n}=2 \pi n / N \quad n=1,2 \ldots N
\end{aligned}
$$

where maximum speed and turning rate are in the right side of Eqn.2. $N$ are the points to approximate a circle.

\subsection{Obstacle avoidance constraints}

As mentioned before, the optimization considers the avoidance of rectangular obstacles, which is modeled by the following constraints in Eqn.3:

$$
\begin{aligned}
& x(t) \leq x^{k}{ }_{\text {min }}+M \delta_{1}{ }^{k}(t) \\
& y(t) \leq y^{k}{ }_{\text {min }}+M \delta_{2}{ }^{k}(t) \\
& x(t) \geq x^{k}{ }_{\text {max }}+M \delta_{3}{ }^{k}(t) \\
& y(t) \geq y^{k}{ }_{\text {max }}+M \delta_{4}{ }^{k}(t)
\end{aligned}
$$

Where $k$ identifies the obstacle, $\left(x_{\min }, y_{\min }\right)$ is its lower left corner and $\left(x_{\max }, y_{\max }\right)$ its upper right corner, $M$ is an upper bound for $x(t)$ and $y(t)$, and $\delta_{j}^{k}$ indicator variables. The $j^{\text {th }}$ condition is then relaxed if $\delta_{j}^{k}=1$, and activated if $\delta_{j}^{k}=0$. In Eqn.3 at least one constraint should be active, and hence we impose that in Eqn. 4 .

$$
\sum_{j=1}^{4} \delta_{j}^{k}(t) \leq 3
$$

\subsection{Arrival constraints}

To force the UAV to get the final position the following constraints (Eqn.5) must be satisfied $\forall t$ :

$$
\begin{aligned}
& x(t)-x_{f} \leq M\left(1-\lambda_{t}\right) \\
& y(t)-y_{f} \leq M\left(1-\lambda_{t}\right) \\
& x(t)-x_{f} \geq-M\left(1-\lambda_{t}\right) \\
& y(t)-y_{f} \geq-M\left(1-\lambda_{t}\right) \\
& t_{\text {arrival }} \geq \sum_{0}^{T} t \cdot \lambda_{t}
\end{aligned}
$$

where $\left(x_{f}, y_{f}\right)$ is the target point, $\lambda_{t}$ is a binary indicator variable, and $t_{\text {arrival }}$ is the time to be minimized in the objective function.

\subsection{Objective function}

The trajectory will be optimized respect to a double objective: flight time and acceptable threat (Eqn.6):

$$
J=\mu_{1} t_{\text {arrival }}+\mu_{2} D\left(x, y, v_{x}, v_{y}\right)
$$

where $D$ is a nonlinear radar detection function modeled in section $3 . \mu_{1}$ and $\mu_{2}$ are weights which consider the importance of each objective concerning to a particular mission.

\section{Radar detection model}

In this paper we consider a simplified model for minimizing the detection risk of an UAV by radars with variable $R C S$. The detection risk $D$ is proportional to the UAV's $R C S$, reciprocal to the fourth power of the distance between the UAV and the radar, and independent of the UAV speed (Figure 2).

$$
D=R C S / r_{R}^{4}
$$

The UAV is considered to be an ellipsoid with the axis of ellipsoid symmetry determining the direction of the UAV trajectory [2]. The UAV's RCS exposed to the radar is proportional to the area of the ellipsoid's projection onto the plane orthogonal to vector $\boldsymbol{r}_{\boldsymbol{R}}$, as shown in Figure 2. Therefore, the $R C S$ is given by:

$$
R C S=\sigma \cdot S
$$

where $\sigma$ depends on the radar technical characteristics, and $S$ is the ellipsoid's projection given by Eqn.9.

$$
S=\pi \sqrt{a^{2} \sin ^{2} \beta+b^{2} \cos ^{2} \beta}
$$

$\beta$ is the angle between vectors $\boldsymbol{r}_{\boldsymbol{R}}$ and $\boldsymbol{v}$ (velocity), and the ellipsoid is defined by semi-axis $a$ and $b$.

As the altitude is fixed $(h), \beta$ will be a function of $x$, $y, v_{x}$, and $v_{y}$, then the detection risk might be expressed as in Eqn.10, such that:

$$
\begin{aligned}
& D\left(x, y, v_{x}, v_{y}\right)= \\
& \sqrt{\frac{\left(h v_{y}\right)^{2}+\left(h v_{x}\right)^{2}+\left(v_{x} y-v_{y} x\right)^{2}+\left(\frac{b^{2}}{a^{2}}\right)\left(v_{x} x+v_{y} y\right)^{2}}{\left(v_{x}^{2}+v_{y}^{2}\right)\left(x^{2}+y^{2}+h^{2}\right)^{9}}}
\end{aligned}
$$




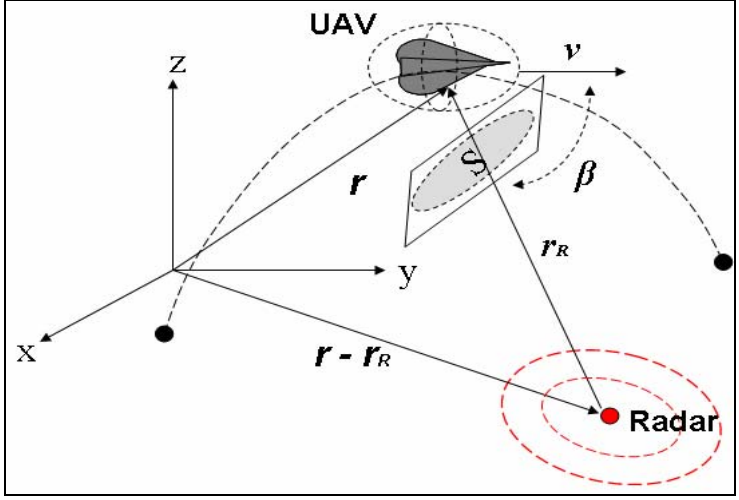

Figure 2. Layout of an UAV under radar detection risk.

\subsection{Linearization of radar detection model}

In order to include the detection risk function (Eqn.10) in a MILP framework, it has been approximated by a piecewise first order linearization $D_{L}$ showed in Eqn.11.

$D_{L}=\sum_{i}\left(a_{i} x(t)+b_{i} y(t)+c_{i} v_{x}(t)+d_{i} v_{y}(t)+e_{i}\right) \delta_{i}(t)$

where $a_{i}, b_{i}, c_{i}, d_{i}$, and $e_{i}$ are the coefficients of the $i^{\text {th }}$ hyper-plane inside the $i^{\text {th }}$ domain, $\delta_{i}(t)$ is a binary indicator variable identifying the domain $\left[l x_{i}, l y_{i}, v_{x}, v_{y}\right]$ $\left[u x_{i}, u x_{i}, v_{x}, v_{y}\right]$ that must take value 1 only in this domain, so must be constrained to:

$\sum_{i=1}^{p} l x_{i} \delta_{i} \leq x(t) \leq \sum_{i=1}^{p} u x_{i} \delta_{i}$

$\sum_{i=1}^{q} l y_{i} \delta_{i} \leq y(t) \leq \sum_{i=1}^{q} u y_{i} \delta_{i}$

$\sum_{i=1}^{p \cdot q} \delta_{i}(t)=1$

To change the positive and negative range of $x(t)$, $y(t), v_{x}(t), v_{y}(t)$ to the $[0,1]$ interval, we apply the following change of variables:

$x(t)=x^{b}(t)\left[u_{x}-l_{x}\right]+l_{x}$

$y(t)=y^{b}(t)\left[u_{y}-l_{y}\right]+l_{y}$

$v_{x}(t)=v_{x}^{b}(t)\left[u_{v x}-l_{v x}\right]+l_{v x}$

$v_{y}(t)=v_{y}^{b}(t)\left[u_{v y}-l_{v y}\right]+l_{v y}$

$0 \leq x^{b}(t), x^{b}(t), v_{x}^{b}(t), v_{y}^{b}(t) \leq 1$

where $u$ and $l$ are upper-bounds and lower-bound of $x$, $y, v_{x}$ and $v_{y}$, respectively.

To linearize the products $V^{b}(t) \delta_{i}(t)$ (where $V^{b}(t)=$ $\left.x^{b}(t), y^{b}(t), v^{b}(t), v^{b}(t)\right)$ of a binary variable $\delta_{i}(t)$ and a continuous variable $V^{b}(t)$, we replace $V^{b}(t) \delta_{i}(t)$ for

$$
W_{i}^{b}(t)=x_{i}^{b}(t), y_{i}^{b}(t), v_{x, i}^{b}(t), v_{y, i}^{b}(t)
$$

and impose the following constraints[11]:
$W_{i}^{b}(t) \leq \delta_{i}(t)$

$W_{i}^{b}(t) \leq V_{i}^{b}(t)$

$W_{i}^{b}(t) \geq V_{i}^{b}(t)+\delta_{i}(t)+1$

So, piecewise detection risk function will be:

$D_{L}=\sum_{k} A_{k} x_{k}^{b}+B_{k} y_{k}^{b}+C_{k} v_{x, k}^{b}+D_{i j} v_{y, k}^{b}+E_{k} \delta_{k}$

where $A_{k}=a_{i j}\left(u_{x}-l_{x}\right), \ldots$

The coefficients are calculated by selecting five points over the hyper-surface of $D$, and solving the corresponding system of equations. Figure 3 show the hyper-planes approximation table for $D_{L}\left(x, y, v_{x}, v_{y}\right)$

\begin{tabular}{|c|c|c|c|c|c|c|c|c|c|c|}
\hline \multirow{3}{*}{$\begin{array}{c}D_{L}\left(x, y, v_{x}, v_{y}\right) \\
y\end{array}$} & \multicolumn{10}{|c|}{$x$} \\
\hline & \multicolumn{5}{|c|}{$\left[l x_{l}, u x_{l}\right]$} & \multicolumn{5}{|c|}{$\left[l x_{2}, u x_{2}\right]$} \\
\hline & $a$ & $b$ & $c$ & $d$ & $e$ & $a$ & $b$ & $c$ & $d$ & $e$ \\
\hline$\left[l y_{1}, u y_{1}\right]$ & $a_{11}$ & $b_{11}$ & $c_{11}$ & $d_{11}$ & $e_{11}$ & $a_{12}$ & $b_{12}$ & $c_{12}$ & $d_{12}$ & $e_{12}$ \\
\hline$\left[l y_{2}, u y_{2}\right]$ & 21 & $b_{21}$ & $c_{2}$ & $d_{21}$ & $e_{2}$ & $a_{22}$ & $b_{2}$ & & & \\
\hline
\end{tabular}

Figure 3. Hyper-planes table for $D\left(x, y, v_{x}, v_{y}\right)$.

For example, the first hyper-plane corresponding to the first range has the following values:

$\left[l x_{1}, u x_{l}\right]=[0 \mathrm{Km}, 1 \mathrm{Km}] ;\left[l y_{1}, u y_{1}\right]=[0 \mathrm{Km}, 1 \mathrm{Km}]$

$a_{11}=-2.246831 e-6 ; b_{11}=-2.246831 e-6$

$c_{11}=-6.910890 e-7 ; d_{11}=6.910890 e-7$;

$e_{11}=1.001382 e-4$

Figure 4 shows the original function $D$ and Figure 5 the piecewise approximation $D_{L}$. The relative error of $D$ achieved with this approximation was under $5 \%$.

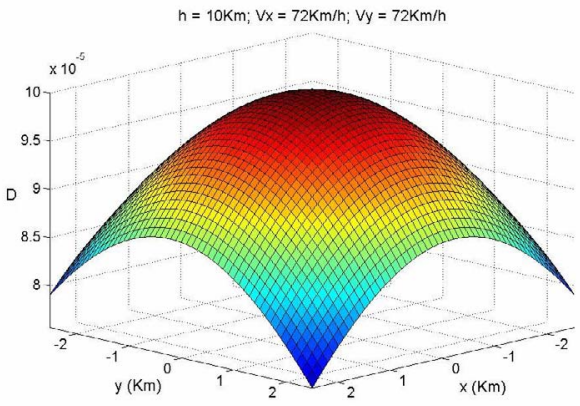

Figure 4. Radar detection function $D(x, y)$.

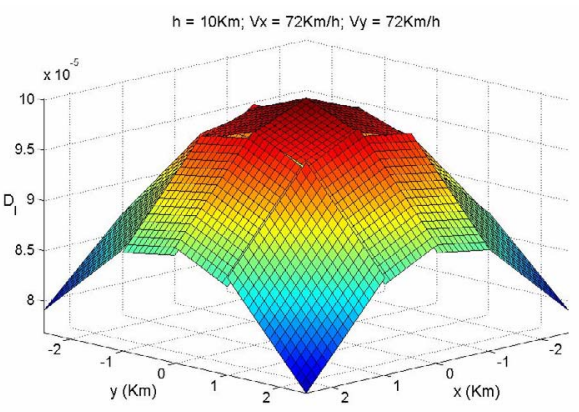

Figure 5. Linearized radar function $D_{L}(x, y)$. 


\section{Implementation and results}

The OPL modeling language has been used for representing the optimization model showed in this paper. The OPL model is then translated to be solved with ILOG CPLEX 9.0. The results have been obtained on a $2.4 \mathrm{GHz}$ Intel Xeon computer with $2 \mathrm{~GB}$ of RAM, running under Windows Server 2003 operating system. In the experiments we have used the following data: $v_{\max }=144 \mathrm{Km} / \mathrm{h}$, discrete time interval $=20 \mathrm{~s}, h=$ $10 \mathrm{Km}, b / a=0.5$, Obstacle $_{\text {Size }}=6.0 \mathrm{Km} \times 2.5 \mathrm{Km}$, starting point $(-4 \mathrm{Km},-4 \mathrm{Km})$, target point $(1.1 \mathrm{Km}$, $1.1 \mathrm{Km})$ and range bounds: $l_{x}=-2.5, l_{y}=-2.5, u_{x}=2.5$, $u_{y}=2.5, l_{v x}=-144, l_{v y}=-144, u_{v x}=144, u_{v y}=144$.

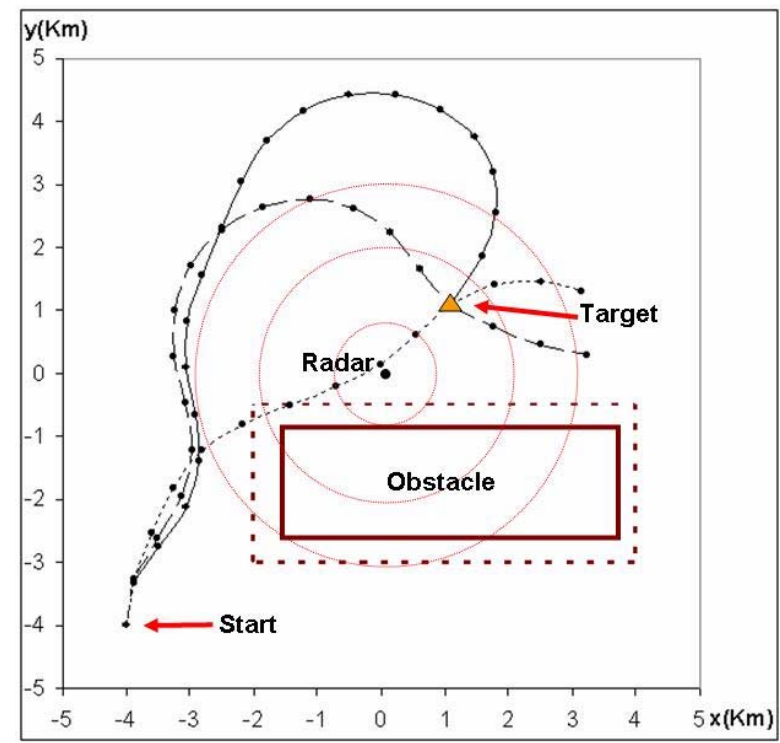

Figure 6. Comparison of three trajectories with different risk (dotted line $\mu_{1}=1.0, \mu_{2}=$ 0.0 ; dashed line $\mu_{1}=1.0, \mu_{2}=2.7 e 4$; solid line $\mu_{1}=1.0, \mu_{2}=2.8 \mathrm{e} 4$ ).

The UAV tries to avoid the radar detection by maintaining the biggest possible distance, compatible with the values $\mu_{1}$ and $\mu_{2}$, and controlling the RCS it presents to the radar. The trajectories plotted in Figure 6 shows that the UAV does not fly directly to the target, and when a higher risk of detection is even accepted, the UAV will use a more direct and risky trajectory $\left(\mu_{1}=1, \mu_{2}=2.7 \mathrm{e} 4\right)$. It can be observed that when the UAV is next to the target and the admitted risk is low $\left(\mu_{1}=1, \mu_{2}=2.8 \mathrm{e} 4\right)$, its trajectory tries to approach radially to the radar, minimizing its RCS. Over a no radar zone $\left(\mu_{2}=0\right)$ the flying trajectory goes directly to the target.

\section{Conclusions and future works}

We have developed a model for UAV trajectory optimization under radar detection with variable RCS. Approximating the continuous risk function with hyper-planes using integer 0-1 variables, an efficient MILP formulation has been possible for the whole system. This has allowed exploiting the current MILP powerful commercial packages such as CPLEX. This approach can be extended to the case of $n$ radars. This case the global risk function is the sum of $n$ risk functions.

We are extending this approach to the case of a radar tabulated model using two tables, $T_{R C S}(\theta, \phi)$ for $R C S$ as a function of azimuth $\theta$ and elevation angle $\phi$, and $P_{D}(R C S, r)$ for the probability of detection as function of $R C S$ an range $r$.

\section{Acknowledgments}

This research was funded by the Community of Madrid, project COSICOLOGI S-0505/DPI-0391.

\section{References}

[1] P.T. Kabamba, S.M. Meerkov and F.H. Zeit Optimal Path Planning for Unmanned Combat Aerial Vehicles to Defeat Radar Tracking, Journal of Guidance, Control, and Dynamics, pp. 279-288, 2006

[2] R. Murphey, S. Uryasev, and M.Zabarankin Trajectory Optimization in a Threat Environment, Research Report 2003-9, Department of Industrial \& Systems Engineering, University of Florida, 2003.

[3] A. G. Richards, Trajectory Optimization using Mixed-Integer Linear Programming, Master's thesis, Massachusetts Institute of Technology, June 2002.

[4] A. Richards and J. P. How, Aircraft Trajectory Planning with Collision Avoidance Using Mixed Integer Linear Programming, Proceedings of the American Control Conference, Anchorage, 2002.

[5] P. Van Hentenryck, The OPL optimization programming language. MIT press, Cambridge, 1999.

[6] ILOG, ILOG CPLEX 9.1 User's guide, 2003.

[7] A. Bemporad and M. Morari, Control of systems integrating logic, dynamics, and constraints, Automatica, vol. 35, pp. 407-427, 1999.

[8] J. Bellingham, M. Tillerson, A. Richards, and J. How, Multi-Task Allocation and Path Planning for Cooperating UAVs, Second Annual Conference on Cooperative Control and Optimization, Nov 2001.

[9] J. Bellingham, A. Richards and J. How, Receding Horizon Control of Autonomous Aerial Vehicles, Proc. of American Control Conference, 2002.

[10] J. S. Bellingham, Coordination and Control of UAV Fleets using Mixed-Integer Linear Programming, Master's thesis, MIT, 2002.

[11] H.P. Willians, Model Building in Mathematical Programming, Wiley, 1999. 